\title{
Potassium-Sparing Diuretics in Hypertension
}

\author{
Cristiana Catena, GianLuca Colussi and Leonardo A. Sechi \\ Clinica Medica, Department of Experimental and Clinical Medicine, University of Udine, \\ Italy
}

\section{Introduction}

Abbreviations and acronyms in this chapter.

ENaC: amiloride-sensitive epithelial sodium channel; ROMK: renal outer medullary $\mathrm{K}$ channel; $\mathrm{BK} \mathrm{K}^{+}$: flow-sensitive maxi $\mathrm{K}$ channel; AQP-2: apical aquaporin; $\mathrm{AQP}-3$ and 4: basolateral aquaporin; $11 \beta$-HSD2: $11 \beta$-hydroxysteroid dehydrogenase; NAD: nicotinamide adenine dinucleotide; ROS: reactive oxygen species; CYP11B2: aldosterone synthase; GPR30: G protein coupled receptor; PI3K: phosphatidylinositol 3-kinase; ERK: extracellular signalregulated kinase; NYHA: New York Heart Association; HFPSF: heart failure and preserved systolic function (HFPSF); ACE: angiotensin-converting enzyme; CCB: calcium-channel blockers.

Many clinical studies have reported that cardiovascular morbidity and mortality have a continuous relationship with both systolic and diastolic blood pressures. Although some of these studies have reported that this relationship is steeper for stroke, in some European countries the attributable risk, that is the excess of events due to increased blood pressure, appears to be greater for coronary artery disease (Prospective Studies Collaboration, 2002). Current evidence indicates that both systolic and diastolic blood pressure levels have a continuous and independent relationship with a variety of additional organ complications, including congestive heart failure, renal failure, and peripheral artery disease. The wide prevalence of hypertension in the general population (Wolf-Maier et al. 2003) explains why hypertension has been identified as the first cause of death worldwide by the World Health Organization. This is also why today hypertension is considered as the most important correctable risk factor for cardiovascular diseases.

Recent guidelines have put strong emphasis on the relevance of hypertension-related subclinical organ damage and have prescribed that signs of organ involvement should be sought with great care. In fact, a large body of evidence indicates that hypertensive subclinical organ damage, including left ventricular hypertrophy, microalbuminuria, and thickening of inner vascular layers, is critical in determining the cardiovascular risk of individuals with high blood pressure (The Task Force for the Management of Arterial Hypertension of the European Society of Hypertension and of the European Society of Cardiology, 2007). In patients with high blood pressure, left ventricular hypertrophy has been shown repeatedly to be associated with an increased incidence of cardiovascular events, and similar evidence has been obtained for carotid intima-media thickening and microalbuminuria. Also and most important, prospective studies have demonstrated that reduction in left ventricular mass, intima-media thickness, and urinary protein excretion 
induced by antihypertensive treatment are associated with reduced incidence of cardiovascular events. This indicates that assessment of subclinical organ damage is critical not only for quantification of the cardiovascular risk, but also to monitor the beneficial effects of treatment. Large clinical trials that have evaluated the effects of blockade of the renin-angiotensin system with angiotensin-converting enzyme inhibitors or angiotensin receptor blockers have demonstrated a specific ability of these agents in preventing progression and inducing regression of hypertension-related subclinical organ damage. It must be noticed, however, that a significant percentage of hypertensive patients with left ventricular hypertrophy fail to achieve full regression of left ventricular mass when treated with renin-angiotensin system blockers, a fact that might be ascribed to plasma aldosterone escape from the inhibitory effect of these drugs. This hypothesis received the support of studies in which it was demonstrated that with use of renin-angiotensin system blockers left ventricular mass does not decrease in hypertensive patients with aldosterone escape as opposed to a decline in left ventricular mass that is observed in patients without aldosterone escape.

Several randomized controlled trials have tested the benefits of blood pressure lowering treatment providing undisputable evidence. Also, several meta-analyses that have collected an impressive number of patients have confirmed the evidence of benefits of treatment in event-based trials comparing active treatment with placebo, different active treatments, and more or less intense blood pressure lowering strategies. Findings of all these studies indicate that: a) use of antihypertensive drugs causes significant reductions of cardiovascular morbidity and mortality; b) reduction of cardiovascular risk is comparable in hypertensive patients of different ethnicity; c) the benefit of antihypertensive treatment is evident even in the elderly patients with isolated systolic hypertension; d) relative reduction of risk is greater for stroke (approximately 35\%) than for coronary artery disease (approximately $20 \%$ ). Overall, studies comparing different types of antihypertensive drugs show that differences in the incidence of cardiovascular morbidity and mortality are not relevant in the presence of comparable blood pressure reduction, thus supporting the conclusion that benefits of treatment are largely dependent on blood pressure reduction per se.

Diuretics decrease the total body pool of sodium-chloride and are included among the five major classes of antihypertensive agents that are suitable for the initiation and maintenance of antihypertensive treatment alone or in combination. This is due to the evidence of effective blood pressure lowering and effective prevention of cardiovascular events that has been obtained with thiazide diuretics. Current guidelines indicate congestive heart failure, isolated systolic hypertension, hypertension in blacks, and end-stage renal disease (this for loop diuretics) as conditions favoring use of diuretics versus other antihypertensive drugs. However, side effect of these drugs including decrease in plasma potassium, and increase in plasma glucose, cholesterol, triglyceride, and uric acid are important limiting factors.

Other classes of diuretics that are used in hypertension as monotherapy, but more frequently as combinations, are the so-called potassium-sparing diuretics. These include a group of drugs that act with different mechanisms at the distal tubular site of the nephron where the mineralocorticoid receptors are expressed and mediate the tubular effects of aldosterone and other mineralocorticoid hormones. The aldosterone-sensitive tubular site of the distal tubule handles only a small amount of sodium chloride and this is why the natriuretic effect of agents acting at this site is relatively modest. However, this tubular site contributes greatly to regulation of body potassium content and acid-base equilibrium by modulation of exchange of sodium with either potassium or hydrogen ions. 
Aldosterone is a steroid hormone that is secreted by the zona glomerulosa of the adrenal cortex and is involved in regulation of blood pressure exerting its main effects on the distal tubular site of the nephron where it increases water and sodium chloride reabsorption thereby leading to expansion of the extracellular fluid volume. Recent views indicate that, in addition to its renal effects and regulatory role on body water and electrolyte balance, aldosterone acts on a variety of cell types affecting cellular mechanisms that mediate important tissue responses, including hypertrophy and fibrosis. Recent evidence obtained from experimental animal studies indicates that chronic exposure to inappropriately high aldosterone levels or activation of the mineralocorticoid receptors can induce tissue damage in specific organ sites with mechanisms that are independent of blood pressure elevation. These animal studies have demonstrated that tissue damage can be prevented by removal of adrenal glands or administration of aldosterone antagonists.

Spironolactone and eplerenone (Figure 1) are mineralocorticoid receptor antagonists commonly employed to reduce blood pressure, left ventricular hypertrophy, and urinary albumin excretion in patients with essential hypertension or primary aldosteronism. The latter effects occur beyond what is expected from the mere reduction of blood pressure, suggesting that activation of the mineralocorticoid receptor plays a central role in the development of cardiac and renal abnormalities in hypertensive patients. In agreement with

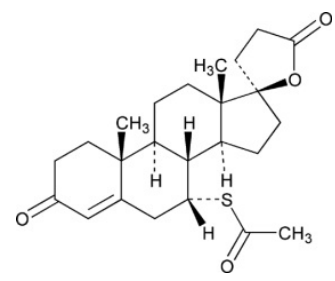

Spironolactone

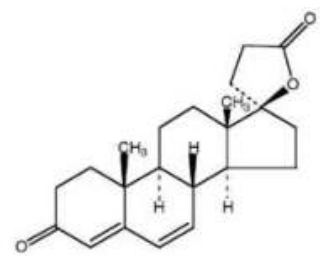

Canrenone

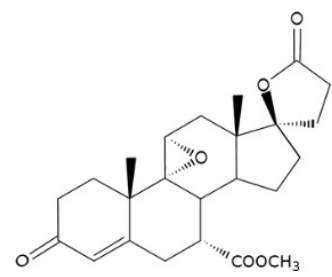

Eplerenone

Fig. 1. Structure of the mineralocorticoid receptor antagonists currently available for clinical use

these findings, currently available evidence strongly supports the notion that aldosterone receptor antagonists are of considerable therapeutic value in patients with systolic heart failure with a cardioprotective effect that is already appreciable in the early functional 
stages. Also, recent evidence suggests that the benefits of aldosterone antagonists in the context of cardiac failure are not restricted to patients with impaired systolic function but can be extrapolated also to patients with diastolic dysfunction. Finally, some studies support the view that mineralocorticoid receptor blockade may exert an antialbuminuric effect in patients with proteinuria, an effect that occurs independent of blood pressure reduction.

The use of classic mineralocorticoid receptor antagonists, however, has been limited by the high incidence of breast engorgement and gynecomastia and the risk of severe hyperkalemia. To overcome these tolerance problems, new aldosterone blockers have been developed (Garthwaite \& McMahon 2004) using two different strategies that include search for non-steroidal antagonists and inhibition of aldosterone synthesis. Inhibition of aldosterone synthesis could have an additional benefit due to blockade of the mineralocorticoid receptor-independent pathways that might account for some of the untoward effects of aldosterone. The new aldosterone blockers are currently having extensive preclinical evaluation, and one of them has passed phase-II trials showing promising results in patients with essential hypertension and primary aldosteronism.

\section{Regulation of potassium excretion at the distal tubular site}

More than $98 \%$ of total body potassium is located inside the cells and homeostatic control of extracellular potassium by the intracellular pool is critical in the regulation of plasma potassium concentration. Plasma potassium levels are maintained stable between 3.5 and 5.0 $\mathrm{mEq} / 1$ despite remarkable variability in potassium intake with meals. This balance is due to mechanisms that operate principally at the renal level and regulate potassium excretion. In normal conditions, daily intake of potassium is entirely eliminated by the body, $90 \%$ by the kidney and $10 \%$ by the intestine. Therefore, changes in body potassium content are physiologically regulated by the kidney that compensates with increased reabsorption in conditions of hypokalemia, and increased secretion in conditions of hyperkalemia. Potassium transport occurs along the entire nephron, but the major role in potassium secretion is played at the distal site by the connecting tubule and cortical collecting duct. In these sites, principal cells are responsible for regulation of sodium reabsorption and via the amiloride-sensitive epithelial sodium channel (ENaC) (Figure 2) with the associated potassium and hydrogen ion excretion. Sodium entry via $\mathrm{ENaC}$ generates an excess of negative charges in the tubular lumen that causes intracellular potassium to leave the cell through the renal outer medullary $\mathrm{K}$ channel (ROMK) and the flow-sensitive maxi-K potassium channel $\left(\mathrm{BK} \mathrm{K}^{+}\right)$ (Figure 2). In addition to distal sodium reabsorption through the $\mathrm{ENaC}$, potassium secretion is therefore dependent on distal tubular flow. Aldosterone has direct influence on potassium secretion, activating sodium transport through activation of the $\mathrm{ENaC}$ and increasing the driving force for potassium secretion into the tubular lumen (Figure 3).

\section{Mechanisms of aldosterone-induced tissue damage}

A growing body of evidence suggests that exposure to inappropriate aldosterone levels for salt status or activation of the mineralocorticoid receptor can produce massive myocardial, vascular, and renal tissue injury with mechanisms that are independent of blood pressure (Marney \& Brown, 2007). Landmark experiments demonstrated that chronic aldosterone infusion causes myocardial fibrosis in rats that are maintained on a high-salt diet. Later on, it was demonstrated that aldosterone-induced myocardial fibrosis is preceded by 
inflammatory changes of perivascular tissue, and that both inflammation and fibrosis can be prevented by administration of mineralocorticoid receptor antagonists or adrenalectomy. Similar evidence was obtained in the kidney of uninephrectomized and stroke-prone spontaneously hypertensive rats in which aldosterone produced intrarenal vascular damage, glomerular injury, and tubulointerstitial fibrosis. Elevated aldosterone also caused aortic fibrosis and hypertrophy in different rat models of hypertension, and administration of eplerenone to hypertensive rats corrected vascular remodeling and fibrosis, suggesting a mineralocorticoid receptor-mediated mechanism.

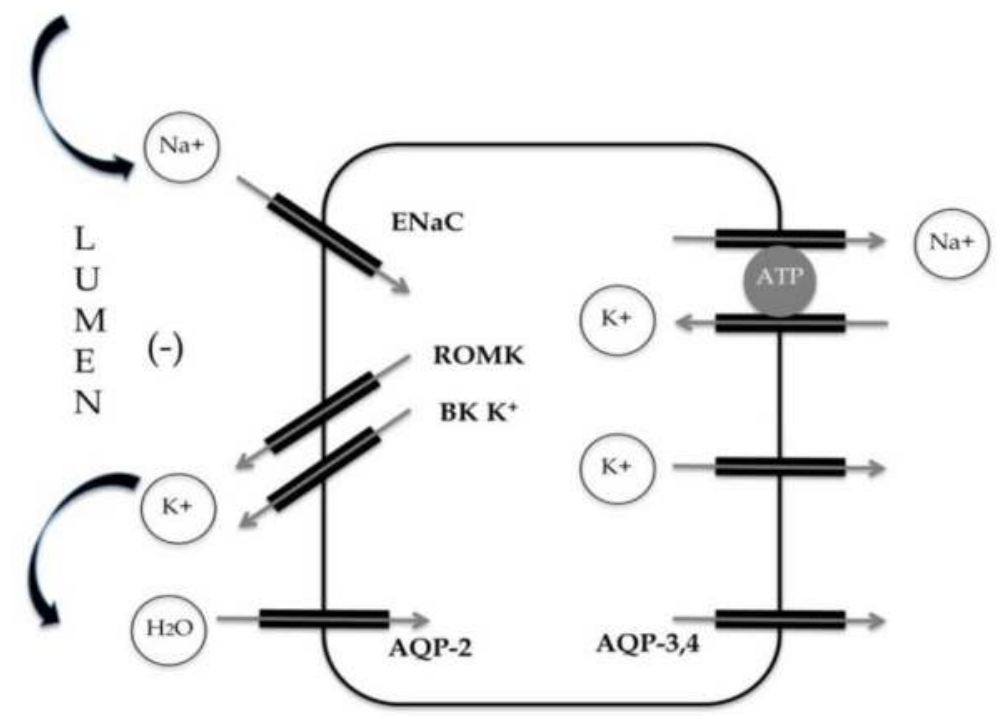

Fig. 2. Sodium, potassium, and water transport in principal cells of connecting tubule and cortical collecting duct. ENaC: amiloride-sensitive epithelial sodium channel; ROMK: renal outer medullary $\mathrm{K}$ channel; $\mathrm{BK} \mathrm{K}^{+}$: flow-sensitive maxi $\mathrm{K}$ channel; AQP-2: apical aquaporin; AQP-3 and 4: basolateral aquaporin.

All these studies consistently indicate that aldosterone causes tissue damage only in the context of inappropriate salt status. It was suggested that untoward effects of high-salt intake are largely dependent on activation of mineralocorticoid receptors and that this activation might reflect increased oxidative stress. Mineralocorticoid receptors are found in epithelial and nonepithelial tissues with high affinity for aldosterone and glucocorticoid hormones, such as cortisol and corticosterone. Under physiological conditions, the majority of mineralocorticoid receptors in nonepithelial tissues are occupied by greater concentrations of cortisol, whereas in epithelial tissues, binding of cortisol to receptors is prevented by $11 \beta$-hydroxysteroid dehydrogenase (11 $\beta$-HSD2), the enzyme that converts cortisol to the receptor-inactive cortisone. In addition to the conversion of cortisol to cortisone, activity of $11 \beta-H S D 2$ generates NADH from NAD and produces changes in the intracellular redox potential that might, in turn, inactivate the glucocorticoid-receptor complex. 11 $\beta$-HSD2 is not present in nonepithelial tissues including the heart, but in such tissues, changes of the intracellular redox potential can result from generation of reactive oxygen species (ROS) and thereby affect the activity of the mineralocorticoid receptor. 


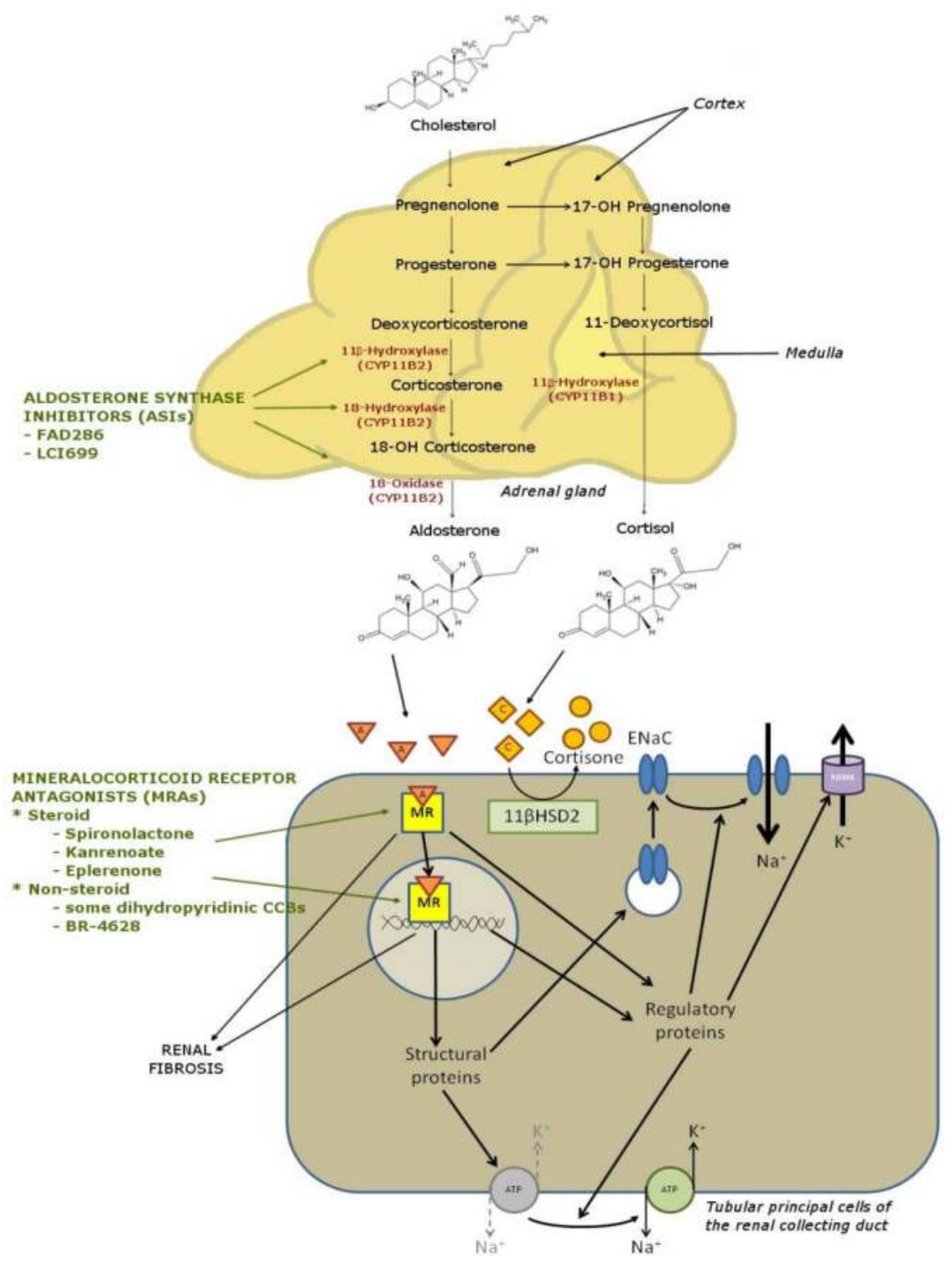

Fig. 3. Aldosterone (A) and cortisol (C) synthesis in the adrenal cortex and mechanisms of aldosterone action in the principal cells of the collecting duct. Aldosterone and cortisol originate from the metabolic conversion of cholesterol in the adrenal cortex. Finals steps of this conversion involve the cytochrome P450 enzymes CYP11B2 and CYP11B1 for the synthesis of aldosterone and cortisol, respectively. Aldosterone synthase inhibitors selectively block CYP11B2 and reduce aldosterone levels. In tubular cells, aldosterone activates the mineralocorticoid receptor and thereby induces structural and regulatory proteins that increase the activity of the epithelial sodium channel $(\mathrm{ENaC})$, the renal outer 
medullary potassium channel (ROMK), and the sodium/potassium ATPase pump. The net effect is sodium reabsorption in the bloodstream and potassium excretion in urine. Steroidal or non-steroidal mineralocorticoid receptor antagonists block the mineralocorticoid receptor activation by aldosterone. Inappropriate activation of mineralocorticoid receptors by cortisol is inhibited by its conversion to the receptor-inactive cortisone by the $11 \beta$-hydroxysteroid dehydrogenase (11 $\beta$ HSD2).

In vitro experiments have demonstrated that changes in the redox potential of cardiomyocytes by exposure to oxidized glutathione turn cortisol from being a receptor antagonist to an agonist. More recently, it has been demonstrated that aldosterone itself induces changes in the intracellular redox potential in diverse cell types through an activation of the NOX1 catalytic subunit of NAD $(\mathrm{P}) \mathrm{H}$ oxidase. This aldosterone-dependent change in the redox potential is amplified by exposure to high concentrations of salt leading to increased production of ROS and thereby to cellular and tissue injury.

In fact, ROS are responsible for apoptosis of cardiomyocytes and for mesangial cell proliferation and matrix expansion in glomeruli of rats. In vascular tissues a slight increase in sodium concentration in the presence of aldosterone affects the biomechanical properties of the endothelial cells leading to cell swelling and cell stiffening (Figure 4). Both these effects are blunted by amiloride (a selective $\mathrm{ENaC}$ inhibitor) or spironolactone demonstrating the involvement of a mineralocorticoid receptor-dependent activation of $\mathrm{ENaC}$ pathway also in the vascular tissue (Figure 4). Furthermore, the analysis of gene expression profiling of vascular tissues demonstrated that, in the context of an enhanced oxidative stress, aldosterone can stimulate the expression of several pro-atherogenic genes and this expression can be inhibited by mineralocorticoid receptor antagonists.

Thus, in addition to the well-known effects of salt loading on epithelial swelling, vascular stiffening and blood pressure increase, some effects of salt loading might depend on mineralocorticoid receptor activation and reflect, in different tissues, impairment of $11 \beta-$ HSD2 activity and/or increased oxidative stress, both mechanisms possibly leading to changes in the intracellular redox state. The distinction of these effects of salt from those generated at the tissue level by elevated aldosterone is complex and even genetic manipulations could not help in the understanding of their respective roles. In fact, both cardiac overexpression of the mineralocorticoid receptor and cardiac-specific induction of aldosterone production do not cause cardiac fibrosis, whereas fibrosis results from knockdown of the cardiac receptor by the use of antisense mRNA.

Although the use of mineralocorticoid receptor antagonists can inhibit or reduce aldosterone effects, several rapid actions of aldosterone on vascular tissue, such as regulation of vascular tone are, at least in part, independent of mineralocorticoid receptor blockade. Therefore, a mineralocorticoid receptor-independent pathway and the existence of a new aldosterone receptor have been hypothesized (Figure 4). Recently, it has been shown that in vascular smooth muscle aldosterone action is linked to a mineralocorticoid receptor-independent pathway that is mediated by the G protein coupled receptor (GPR30) (Figure 4). This pathway involves phosphatidylinositol 3-kinase (PI3K) and the extracellular signalregulated kinase (ERK). These findings rise new questions on the role of aldosterone-related and mineralocorticoid receptor-independent pathways in causing tissue injury. 


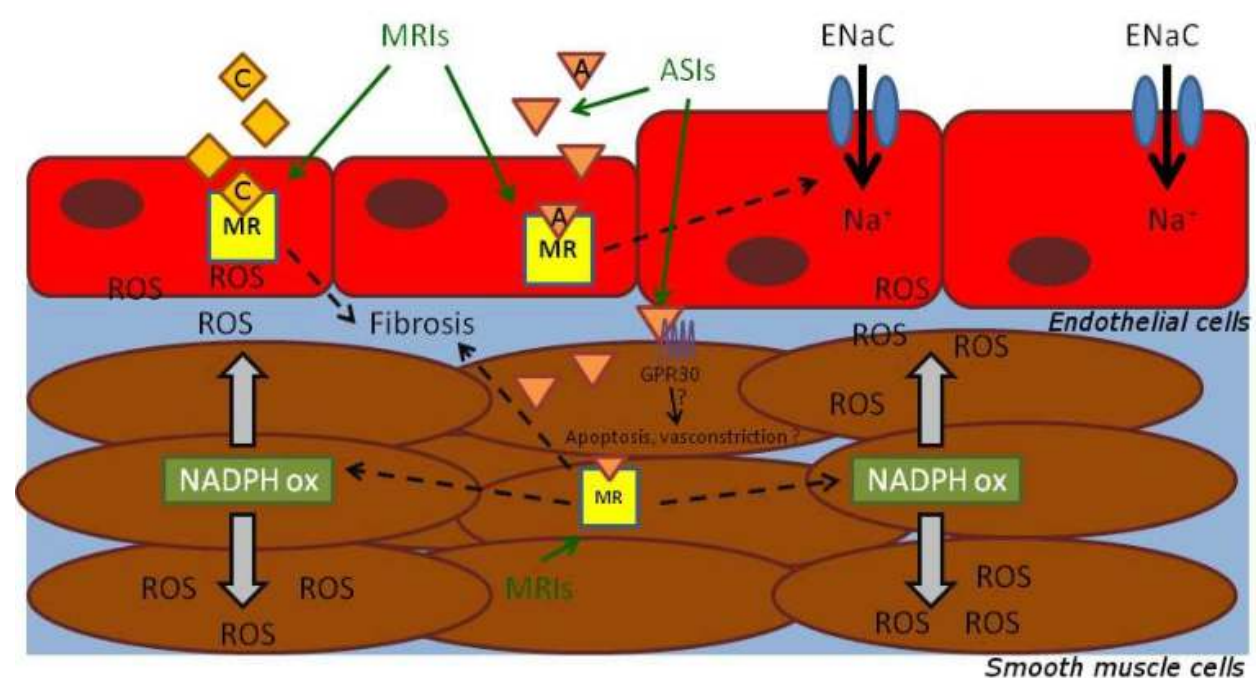

Fig. 4. Effect of aldosterone (A) on blood vessels. Aldosterone binds to mineralocorticoid receptors (MR) increasing generation of reactive oxygen species (ROS) and induces endothelial cell swelling and stiffening via a mineralocorticoid receptor-dependent pathway involving the NADPH oxidase and epithelial sodium channel, respectively. Both effects are blocked by mineralocorticoid receptor antagonists. Increased oxidative stress turns cortisol (C) to be an agonist rather than an antagonist of mineralocorticoid receptor. Aldosterone might exert its deleterious cardiovascular effects also through a mineralocorticoid receptor-independent pathway involving the G protein coupled receptor (GPR30) that can induce vascular smooth muscle cell apoptosis and inappropriate vasoconstriction. The pathophysiological role of these "non-genomic" effects of aldosterone needs to be further explored.

\section{Spironolactone}

Spironolactone (Figure 1) was synthesized in the Searle Laboratories in 1958 and its aldosterone blocking activity was discovered in 1959 (Cella et al., 1959). The molecule was approved for clinical use in 1962 and has been used for many decades in the treatment of primary aldosteronism, ascites associated with portal hypertension, and congestive heart failure. It has been used also for the treatment of primary hypertension (De Gasparo et al., 1987).

\subsection{Metabolism}

Spironolactone is a well-absorbed (approximately 65\%), lipid-soluble, and highly protein bound steroidal aldosterone antagonist. It is largely metabolized in a hepatic first-pass with a high degree of enterohepatic cycling and a half-life of 1.6 hours, as such. In fact, spironolactone is transformed to either 7-thiomethylspirolactone or canrenone, two active metabolites that account for much of its pharmacological activity. Canrenone has a 20-hour half-life, but this is prolonged in patients with cardiac insufficiency. Spironolactone is also an inducer of microsomal drug metabolizing enzymes in the liver. The onset of action of spironolactone is slow, with a peak response that is reached approximately 48 hours after 
the first oral dose. Spironolactone is moderately more potent than eplerenone in blocking mineralocorticoid receptors. Spironolactone remains active when renal function is impaired because it reaches its site of action independent of glomerular filtration. This accounts for the risk of hyperkalemia observed in patients with chronic kidney disease and in patients with congestive heart failure and impaired renal function.

\subsection{Dosing}

The recommended oral dosing range of spironolactone is from 12.5 to $250 \mathrm{mg}$ once or twice a day in primary hypertension and other disease conditions in which the use of this agent is indicated.

\subsection{Clinical use}

Spironolactone is a medication that has been used for more than 50 years to treat hypertension, edema, primary aldosteronism, and, more recent evidence indicates benefits also in patients with congestive heart failure and proteinuria.

Almost a decade ago, a landmark trial investigated the effects of spironolactone in patients with functional class III-IV systolic heart failure, showing a significant decrease in the mortality rate as compared to patients who received placebo on top of conventional treatment. The Randomized Aldactone Evaluation Study (RALES) (Pitt et al., 1999) was conducted in patients with New York Heart Association (NYHA) class III-IV heart failure who were treated with spironolactone. More recently, it has been suggested that the benefits of spironolactone in the context of cardiac failure are not restricted to patients with impaired systolic function and some studies have tested the effects of spironolactone in patients with heart failure and preserved systolic function (HFPSF). Edwards et al. reported improved diastolic function parameters with use of spironolactone in 112 patients with stage 2-3 chronic renal failure and HFPSF who were included in the Chronic Renal Impairment in Birmingham (CRIB II) study. In this study, the effects of spironolactone on left ventricular function and circulating markers of collagen turnover were compared with those of placebo. After 40 weeks, spironolactone improved significantly markers of left ventricular relaxation and attenuated significantly the increase in aminoterminal propeptide of type-III procollagen that was observed with placebo. This and other studies on HFPSF suggest a possible benefit of spironolactone also on this subtype of cardiac insufficiency. Notably, all these studies have employed doses of spironolactone (from 25 to $50 \mathrm{mg} /$ day) that did not lower blood pressure suggesting that the cardioprotective effects of spironolactone occurs independent of the blood pressure-related hemodynamic load to the heart. Taken together, the findings obtained in the studies that have tested the effects of spironolactone in heart failure provide indirect evidence of untoward effect of aldosterone on the heart.

Many studies have reported a beneficial effect of blockers of the renin-angiotensinaldosterone system in slowing down progression of renal disease, but the relative contributions of angiotensin II versus aldosterone have been dissociated only recently in animal studies. Clinical studies have supported the view that mineralocorticoid receptor blockade may exert an antialbuminuric effect in patients with proteinuria. In patients with diabetic nephropathy, it was shown that the antiproteinuric effect of angiotensin-converting enzyme (ACE) inhibitors reverts to baseline in patients who manifest aldosterone escape 
and that spironolactone combined with an ACE inhibitor results in an additional decrease in albuminuria (Sato et al., 2003). Also, spironolactone effectively reduced proteinuria in patients with idiopathic chronic glomerulonephritis previously treated with either ACE inhibitors or angiotensin receptor blockers (Bianchi et al., 2005). Another, more recent, study has shown that spironolactone added to an ACE inhibitor or an angiotensin receptor blocker reduces albuminuria in patients with type 2 diabetes and nephropathy. Collectively, these observations suggest that the mechanisms of the antialbuminuric effect of spironolactone are independent of blood pressure reduction and occur on top of those of other blockers of the renin-angiotensin system.

\subsubsection{Essential hypertension}

The anti-hypertensive effects of spironolactone have been overviewed in a recent metaanalysis that has included five cross-over studies and one randomized controlled trial with a total of 179 essential hypertensive patients that were followed from 4 to 8 weeks (Batterink et al., 2010). This meta-analysis showed that spironolactone decreases systolic and diastolic blood pressure by 20 and $7 \mathrm{mmHg}$, respectively, but this effect is reached with doses between 100 and $500 \mathrm{mg}$ per day. With these doses, the risk of hyperkalemia is an important limitation and this is why use of spironolactone in the treatment of essential hypertension has been limited to combination with other types of diuretics. The dose of $25 \mathrm{mg} /$ day did not change either systolic or diastolic blood pressure. None of the studies that were included in this meta-analysis reported results for hard endpoints such as mortality and major cardiovascular events. Therefore, at present there is no evidence that use of spironolactone decreases the risk of cardiovascular disease.

Despite the effects of spironolactone on blood pressure in essential hypertensive patients are modest and there is no demonstration that spironolactone protects from cardiovascular events, possible benefits on subclinical hypertensive organ damage that might be obtained even with the lower doses of the drug should be considered. Some small studies conducted in patients with hypertension-induced left ventricular hypertrophy have reported that addition of spironolactone to blockers of the renin-angiotensin system increases the effects on left ventricular mass reduction. The effects of an ACE-inhibitor alone or an ACE-inhibitor plus spironolactone ( $25 \mathrm{mg} /$ day) on blood pressure and left ventricular mass changes were compared in essential hypertensive patients with left ventricular hypertrophy. Left ventricular mass index decreased in both treatment groups, but the extent of reduction was significantly greater in patients who were treated with the combination of the ACE-inhibitor and spironolactone. Similarly, the effects of candesartan $(8 \mathrm{mg} /$ day) alone or combined with spironolactone $(25 \mathrm{mg} /$ day) were tested in patients with hypertension and different patterns of left ventricular geometry. Changes in blood pressure did not differ between the two groups, whereas only the combination of candesartan and spironolactone decreased left ventricular mass with a change that was significant only in patients with concentric hypertrophy. In another study, 30 hypertensive patients with impaired diastolic function were randomized to receive either $25 \mathrm{mg}$ /day of spironolactone or placebo for 6 months. Peak systolic strain and cyclic variation of integrated backscatter were improved by spironolactone with significant differences with patients treated with placebo. Thus, current evidence indicates that spironolactone could have a considerable place in the treatment of essential hypertensive patients with left ventricular hypertrophy and/or diastolic dysfunction. 


\subsubsection{Resistant hypertension}

Several clinical case reports suggested that spironolactone can be useful in the treatment of resistant hypertension and, in particular, in hypertension associated to obesity or obstructive sleep apnea syndrome. One controlled and several non-controlled studies have confirmed that addition of $25-50 \mathrm{mg}$ of spironolactone to current treatment effectively reduces blood pressure in patients with resistant hypertension. In the ASPIRANT trial, 117 patients with resistant hypertension were randomized to treatment with spironolactone or placebo in a double-blind protocol. The trial was prematurely stopped after the first interim analysis because of a significant reduction of systolic blood pressure in patients taking spironolactone as compared to those taking placebo. Notably, the average BMI of the study population in this trial was 32.3 clearly indicating that patients were either obese or overweight. In the prospective, uncontrolled study of Souza et al., 175 patients with resistant hypertension were treated with $25-100 \mathrm{mg} /$ day of spironolactone and, after a median interval of 7 months, 24-hour systolic and diastolic blood pressure decreased by $16 \mathrm{~mm} \mathrm{Hg}$ and $9 \mathrm{~mm} \mathrm{Hg}$, respectively. The baseline characteristics of these patients showed again that they were either overweight or obese and had high prevalence of diabetes, dyslipidemia, left ventricular hypertrophy, and previous cardiovascular diseases. In the ASCOT-BPLA trial, patients who took spironolactone as fourth line therapy because of resistant hypertension were analyzed retrospectively. In these patients, spironolactone at a median dose of 25 $\mathrm{mg}$ /day significantly reduced blood pressure by $21.9 / 9.5 \mathrm{~mm}$. Even in this study, patients with resistant hypertension had significantly higher body mass index, systolic blood pressure, and prevalence of diabetes and left ventricular hypertrophy than patients who were not resistant to treatment. Very similar results were reported in other studies conducted on patients of different geographical areas.

The beneficial effect of spironolactone in patients with resistant hypertension is currently unexplained, although this effect suggests a substantial contribution of aldosterone to maintenance of increased blood pressure despite of treatment. Inappropriate secretion of aldosterone has been reported in hypertensive patients with associated obesity and/or obstructive sleep apnea syndrome. Also, it is well known that aldosterone can escape the inhibitory effects of renin-angiotensin system blockers in patients treated with these drugs, thereby leading to a form of hypertension that is largely aldosterone-dependent. Finally, it cannot be excluded that, at least in some cases, resistance to antihypertensive treatment hides a mild form of primary aldosteronism.

\subsubsection{Primary aldosteronism}

Spironolactone is the drug of choice in the medical treatment of primary aldosteronism in which hypertension is due to an excessive aldosterone secretion from the adrenal gland. In this clinical setting, spironolactone reduces blood pressure, corrects hypokalemia, and reverts cardiac and renal abnormalities as it has been reviewed recently. This was demonstrated in long-term follow-up studies in which treatment with spironolactone reduced cardiovascular and renal events and decreased left ventricular mass and urinary protein excretion in patients with primary aldosteronism (Sechi et al., 2010).

Cardiovascular outcomes were compared in 108 patients with essential hypertension and in 54 patients with primary aldosteronism who were comparable for demographic variables and had comparable risk factors, but greater retrospective incidence of coronary artery 
disease, cerebrovascular events, and sustained arrhythmias (Catena et al. 2008). Patients were followed for an average of 7.4 years after surgical removal of an adrenal adenoma or treatment with spironolactone, with a combined end point including myocardial infarction, stroke, any type of revascularization procedure, and sustained arrhythmias. During followup, blood pressure was comparable in the primary aldosteronism and essential hypertension group, and 10 patients in the former group and 19 in the latter group reached the end point. Actuarial analysis of patients treated with surgery vs. spironolactone did not reveal significant difference in the occurrence of the combined end point. In the same cohort of patients, the outcomes of renal function were investigated by measuring the rates of change of glomerular filtration and albuminuria (Sechi et al., 2006). After an initial decline in creatinine clearance, due to correction of the aldosterone-induced intrarenal hemodynamic adaptation, subsequent decrease of glomerular filtration in patients with primary aldosteronism and essential hypertension were comparable. Urinary albumin losses did not differ between patients with primary aldosteronism and essential hypertension during follow-up. Evaluation of renal outcomes in patients with primary aldosteronism who were treated with surgery or spironolactone did not reveal significant difference. These two studies clearly demonstrate that spironolactone has the same therapeutic value as surgery in the treatment of primary aldosteronism and in the prevention of cardiovascular and renal complications.

In addition to excess cardiovascular and renal events as compared to matched patients with essential hypertension, patients with primary aldosteronism are characterized by cardiac, renal, and metabolic subclinical structural and functional abnormalities (Rossi et al., 2008). A number of cross-sectional cardiac ultrasound studies have reported an excess increase of left ventricular mass in patients with primary aldosteronism as compared to other types of hypertensive disease. In a 7-year echocardiographic study it was demonstrated that patients with primary aldosteronism treated with either surgery or spironolactone have significant and comparable decrease of left ventricular mass, although decrease is significant within the first year only after adrenalectomy (Catena et al., 2007). We have already mentioned the effects of spironolactone on correction of albuminuria. These effects are at least in part related to reversal of an intrarenal hemodynamic adaptation to aldosterone excess with a vasodilatory response that has been demonstrated with intrarenal echo-Doppler examination (Sechi et al., 2009).

\section{Canrenoate}

Canrenone (Figure 1) is one of the two metabolites of spironolactone. It is administered orally as a potassium salt (potassium canrenoate) that is mineralocorticoid receptor-inactive, but it is rapidly transformed to canrenone. Canrenone is water soluble, and this characteristic permits intravenous administration when a rapid effect is desired.

Potassium canrenoate exerts its hypotensive effect approximately one week after the starting dose. Both canrenone and canrenoate are rapidly absorbed (approximately $80 \%$ ) after oral administration. Both agents have important plasma protein binding (approximately 90\%) with small volumes of distribution.

\section{Eplerenone}

Eplerenone (Figure 1) has been synthesized in the attempt to obtain a more selective inhibition of the mineralocorticoid receptors to overcome the side effects due to crossreaction of spironolactone and canrenone with androgen receptors. It was synthesized in the 
Ciba-Geigy laboratories in the mid-80s and was approved in the United States for clinical use in arterial hypertension in 2002.

\subsection{Pharmacology}

Eplerenone was synthesized by replacing the 17-thioacetyl group with a carbomethoxy group in the molecule of spironolactone (Figure 1). The critical feature in the eplerenone molecule however, conferring enhanced mineralocorticoid receptor selectivity is the presence of the epoxide group in the lactone ring. The activity of eplerenone in vitro was assessed in vitro using recombinant steroid receptors. The potency of eplerenone at other steroid receptors was significantly reduced and, unlike previous aldosterone blockers, eplerenone possesses very low activity on the androgen, progesterone, and glucocorticoid receptors.

Oral bioavailability is approximately $95 \%$ and meals have no effect on the extent of absorption. Eplerenone does not undergo relevant metabolic first-pass in the liver neither it induces cytochrome P450 activity, although interactions with drugs that are metabolized by cytochrome P450 are not excluded. The two main metabolites of eplerenone $(6 \beta-\mathrm{OH}$ eplerenone and open lactone ring) are both mineralocorticoid receptor-inactive. The plasma half-life of eplerenone is approximately 5 hours.

The recommended oral dosing range for eplerenone is from 50 to $100 \mathrm{mg}$ once or twice daily in essential hypertension. No correlations between eplerenone disposal and renal function have been found.

\subsection{Clinical use}

Eplerenone has come into the clinical arena in the last decade and has been employed to lower blood pressure in essential hypertensive patients. Similar to spironolactone, recent studies have reported beneficial effects of eplerenone in congestive heart failure and renal disease with proteinuria. The Eplerenone Post-Acute Myocardial Infarction Heart Failure Efficacy and Survival Study (EPHESUS) (Pitt et al. 2003) investigated the effects of eplerenone in postmyocardial infarction patients with severely impaired left ventricular function, showing a significant decrease in the mortality rate as compared to patients who received placebo on top of conventional treatment. Recently, these observations have been extended to patients with milder degrees of cardiac dysfunction in the Eplerenone in Mild Patients Hospitalization and Survival Study in Heart Failure (EMPHASIS-HF) (Zannad et al., 2011) study. In this study, 2737 patients with NYHA class II cardiac insufficiency and left ventricular ejection fraction of less than $35 \%$ were randomized to receive either eplerenone or placebo in addition to conventional treatment. This trial ended prematurely after a median follow-up of 21 months because the composite endpoint of cardiovascular death and hospitalization for heart failure were significantly less frequent in patients who were treated with eplerenone. Thus, eplerenone seems to be beneficial even at the early stages of systolic cardiac failure.

As for spironolactone, the possibility that eplerenone may result beneficial also in patients with HFPSF has been investigated. Forty-four elderly patients with heart failure and left ventricular ejection fraction of more than $45 \%$ were randomized to conventional treatment with or without eplerenone and left ventricular function was reassessed with conventional echocardiography and tissue Doppler imaging at 6 and 12 months. In patients who were treated with eplerenone, deceleration time had a significantly greater decrease than in 
patients on conventional treatment and, after 12 months, the eplerenone-induced improvement of diastolic function was associated with a significantly slower increase in plasma procollagen levels. Both studies on systolic and diastolic heart failure were conducted with doses of eplerenone (from 25 to $50 \mathrm{mg}$ / day) that did not lower blood pressure.

Consistent with previous findings using spironolactone, eplerenone has been demonstrated to confer renal protection independent of its effects on blood pressure. Eplerenone was administered in doses of 50 and $100 \mathrm{mg} /$ day to patients with type 2 diabetes and albuminuria who were already treated with an ACE inhibitor. After 4, 8, and 12 weeks, both doses of eplerenone induced significant reduction of urinary protein losses as compared to placebo, whereas blood pressure levels did not differ during follow-up. Effects of the two doses of eplerenone were comparable (Epstein, 2003).

\subsubsection{Essential hypertension}

Eplerenone has been repeatedly tested in patients with mild to moderate essential hypertension. One uncontrolled open-label study and many randomized controlled trials have documented the antihypertensive effect of eplerenone when compared to either placebo or other classes of antihypertensive agents. Controlled studies have compared the blood pressure lowering effects of eplerenone at doses comprised form 50 to $400 \mathrm{mg}$ /day with those of either placebo, enalapril, losartan, amlodipine, or spironolactone. In these studies, follow-up duration was from 2 to 14 months. In all studies, eplerenone was more effective than placebo in reducing blood pressure, in two studies it was more potent than losartan, and in the remaining studies the hypotensive effects were comparable to those of enalapril, amlodipine, and spironolactone. Similar to spironolactone, none of these studies reported results for hard cardiovascular endpoints including mortality and major cardiovascular events and therefore there is no evidence that eplerenone improves cardiovascular outcomes in hypertensive patients.

Eplerenone has been demonstrated to be beneficial also on hypertension-related subclinical organ damage. In the 4-E Left Ventricular Hypertrophy Study, regression of left ventricular hypertrophy was compared in essential hypertensive patients who were treated with eplerenone, enalapril, or their combination for 9 months. Left ventricular mass index decreased significantly and comparably in patients treated with eplerenone or enalapril, whereas the combination of the two agents showed additive effects on left ventricular mass reduction. In other studies, eplerenone was reported to be more effective than amlodipine, enalapril, or losartan in decreasing urinary albumin excretion.

Despite eplerenone has a satisfactory tolerability and safety profile even at the highest doses that have been clinically tested (200 mg/day), its effects on blood pressure in essential hypertension and primary aldosteronism are inferior to those of spironolactone.

\section{Epithelial sodium channel blockers}

$\mathrm{ENaC}$ blockers are potassium-sparing compounds with weak diuretic properties. Both amiloride and triamterene are treatment of choice in the Liddle syndrome, but are relatively uneffective in essential hypertension when used as monotherapy and can be useful when used in combination with other types of diuretics that cause hypokalemia such as thiazides and loop diuretics. 
No studies have evaluated the effects of ENaC blockers in monotherapy in patients with essential hypertension. Four studies with amiloride and 2 studies with triamterene have evaluated the dose-related blood pressure efficacy of these drugs when added, as a second antihypertensive agent, to hydrochlorothiazide or chlorthalidone (Heran et al., 2010).

\subsection{Amiloride}

Amiloride is a selective blocker of the $\mathrm{ENaC}$. It is actively secreted by cationic carriers in the proximal tubule, reaching its active site at the distal tubule. At this level, amiloride indirectly antagonizes the effects of aldosterone on sodium-potassium exchange leading to increased sodium excretion with relative potassium retention. Amiloride is cleared by the kidney and can accumulate in patients with impaired renal function. Therefore, the dosage should be reduced when the glomerular filtration rate is below $50 \mathrm{ml} / \mathrm{min}$.

No studies have evaluated amiloride as monotherapy in patients with essential hypertension. Amiloride is administered in patients with essential hypertension usually in combination with thiazide diuretics. Amiloride can also be used in patients with primary aldosteronism when spironolactone is not tolerated. Although studies that have tested amiloride as an antihypertensive agents have reported good tolerability, particular caution is required in patients with renal failure or in patients treated with blockers of the reninangiotensin system because of the risk of hyperkalemia.

Data on use of amiloride (from 2.5 to $5 \mathrm{mg}$ /day) in combination with hydrochlorothiazide are insufficient to demonstrate a significant difference between treatment with the combination and hydrochlorothiazide alone. Therefore current evidence does not support any effect of low doses of amiloride on blood pressure. No trials evaluating doses of amiloride higher than $5 \mathrm{mg} /$ day have been performed and therefore a dose-response relationship with the hypotensive effect can not be demonstrated. No effects of amiloride on pulse pressure or blood pressure variability could be demonstrated in the studies that have employed this agent in the treatment of hypertension.

There are no appropriately designed studies that have tested the effects of amiloride in other disease conditions. In primary aldosteronism experiences are mainly anedoctal and the doses of this drug to use in this context have never been defined with precision. No studies have been performed to test the effects of amiloride on subclinical hypertensive organ damage, heart failure, and proteinuria.

\subsection{Triamterene}

Similar to amiloride, triamterene blocks the $\mathrm{ENaC}$ in the distal tubular site of the nephron. Triamterene is incompletely absorbed and is transformed to a sulphate-conjugated metabolite. It gains access to the tubular lumen via a cationic transporter in the proximal tubule. Both triamterene and its metabolite accumulate in patients with renal failure. When used alone, triamterene has little effect on blood pressure. Therefore it is usually employed in combination to compensate for the hypokalemic effects of other diuretics.

No studies have evaluated triamterene as monotherapy in patients with essential hypertension. Data on use of triamterene $(50 \mathrm{mg} /$ day) in combination with chlorthalidone are insufficient to demonstrate a significant difference between treatment with the 
combination and chlorthalidone alone. Therefore current evidence does not support any effect of low doses of triamterene on blood pressure. No data are currently available on the effects on blood pressure of higher doses of triamterene and therefore a dose-response relationship with the hypotensive effect can not be demonstrated. No effects of triamterene on pulse pressure or blood pressure variability could be demonstrated in the studies that have employed this agent in the treatment of hypertension. Tolerability and safety profile of triamterene in the studies that have tested this drug were acceptable.

There are no studies that have tested the effects of triamterene in other disease conditions. In primary aldosteronism experiences are mainly anedoctal and the doses of this drug that should be used in this endocrine disorder have never been specifically defined. No studies have been conducted to investigate the effects of triamterene on subclinical hypertensive organ damage, heart failure, and proteinuria.

\section{New aldosterone blockers}

Promising results with mineralocorticoid receptor antagonists in the treatment of hypertension and prevention of hypertension-related organ damage have prompted the search and possible development of new aldosterone antagonists. Search has followed two main strategies: the first has consisted in the development of non-steroidal antagonists that could overcome the side effects of spironolactone and canrenoate without losing the pharmacological properties of these compounds; the second has aimed at developing drugs that inhibit aldosterone biosynthesis and has resulted in the generation of aldosterone synthase direct inhibitors.

\subsection{Non steroidal mineralocorticoid receptor antagonists}

The first strategy moved its initial steps from the demonstration that some dyhydropyridine calcium-channel blockers (CCBs) exerts also a mineralocorticoid receptor antagonist activity. Researchers in the Pfizer ${ }^{\circledR}$ laboratories reported that nimodipine, felodipine, and nitrendipine block aldosterone-induced receptor activation by competing with aldosterone binding to the receptor "ligand binding domain". The affinity of dihydropyridines for the receptor binding domain is lower than for the L-type calcium channels, indicating that inhibition of mineralocorticoid receptor activity is independent of the effects on calcium channels. The efficacy of dyihydropyridines as mineralocorticoid receptor antagonist is comparable to that of eplerenone, whereas non-dihydropyridine CCBs such as verapamil and diltiazem do not have any inhibitory activity.

\subsection{Aldosterone synthase inhibitors}

The other new class of anti-aldosterone agents includes the selective aldosterone-synthase inhibitors. Aldosterone synthase or CYP11B2 is an enzyme of the cytochrome P450 family with steroid 18-hydroxylase and 18-oxidase properties. It catalyzes the formation of aldosterone from 11-deoxycorticosterone within the zona glomerulosa in the adrenal cortex (Figure 3). CYP11B2 deficiency in humans is characterized by a low/absent aldosterone synthesis in the adrenal cortex, high plasma renin activity and is responsible for a sodiumwasting phenotype associated with retarded growth. Conversely, enhanced activation of CYP11B2 as it is supposed to occur in the polymorphism $344 \mathrm{C} / \mathrm{T}$, is associated with 
increased left ventricular mass and greater risk to develop hypertension. Since CYP11B2 activity is the limiting biochemical step in aldosterone synthesis, its selective inhibition is a good target for prevention of aldosterone untoward effects mediated by both mineralocorticoid receptor-dependent and mineralocorticoid receptor-independent pathways.

At present, only two compounds (both synthesized by Novartis ${ }^{\circledR}$ ) with selective CYP11B2 inhibitory properties have been tested in animal models and, very recently, in the clinical setting. FAD286 is the D-enantiomer of the fadrozole, an aromatase inhibitor developed to treat advanced breast cancer, that reduces aldosterone levels and increases plasma renin activity in rats fed with either low or high sodium diet. In a transgenic rat model of secondary hypertension in which the angiotensinogen gene is overexpressed and circulating angiotensin II levels are increased, oral administration of FAD286 has reduced mortality by 4 times. In transgenic rats treated with FAD286, cardiac hypertrophy, albuminuria, and histologic evidence of glomerular damage were less frequent than in control animals. Despite very minor effects of FAD286 on blood pressure, its effects on organ damage were comparable to those of the angiotensin receptor blocker, losartan.

Following promising preclinical results, the first aldosterone synthase inhibitor, LCI699, was tested in two phase-II clinical trials. LCI699 is similar in structure to FAD286 and it has been developed for human use. LCI699 was administered to 14 patients affected by primary aldosteronism and effects were compared to those of placebo. After 2 weeks of treatment, there was a dose-dependent decrease in plasma aldosterone and an increase in 11deoxycorticosterone, potassium, and adrenocorticotropin levels. Treatment induced mild reduction of $24-\mathrm{h}$ ambulatory systolic blood pressure by $(4.1 \mathrm{~mm} \mathrm{Hg})$ after 4 weeks. More recently, Calhoun et al. have published the first randomized, double-blind, placebo controlled phase-II trial with LCI699 that was conducted in 524 patients with essential hypertension. In this trial different doses of LCI699 have reduced 24-h ambulatory systolic blood pressure, but only the highest dose has reduced also diastolic blood pressure.

\section{Acknowledgments}

This work was supported by a research grant of the Pier Silverio Nassimbeni Foundation.

\section{References}

Batterink, J. et al. (2010). Spironolactone for hypertension. Cochrane Database of Systematic Reviews, Issue.1, CD008169

Bianchi, S., Bigazzi, R. \& Campese, V.M. (2005). Antagonists of aldosterone and proteinuria in patients with CKD: an uncontrolled pilot study. American Journal of Kidney Diseases, Vol.46, pp. 45-51, PMID 15983956

Catena, C. et al. (2007). Long-term cardiac effects of adrenalectomy or mineralocorticoid antagonists in patients with primary aldosteronism. Hypertension, Vol.50, pp. 911918, PMID 17893375

Catena, C. et al. (2008). Cardiovascular outcomes in patients with primary aldosteronism after treatment. Archives of Internal Medicine, Vol.168, pp. 80-85, PMID 18195199

Cella, J., Brown, E.A. \& Burtner, R.R. (1959). Steroidal aldosterone blockers. Journal of Organic Chemistry,Vol.24, pp. 743-748 
De Gasparo, M. et al. (1987) Three new epoxy-spironolactone derivatives: characterization in vivo and in vitro. Journal of Pharmacology and Experimental Therapeutics, Vol.240, pp. 650-656, PMID 2949071

Epstein, M. (2003). Aldosterone receptor blockade and the role of eplerenone: evolving perspectives. Nephrology Dialysis Transplantation, Vol.18: pp. 1984-1992, PMID 13679471

Garthwaite, S.M. \& McMahon E.G. (2004). The evolution of aldosterone antagonists. Molecular and CellularEndocrinology, Vol.217, pp. 27-31, PMID 15134797

Heran, B.S. et al. (2010). Blood pressure lowering efficacy of potassium-sparing diuretics (that block the epithelial sodium channel) for primary hypertension. Cochrane Database of Systematic Reviews, Issue.1, CD008167

Marney, A.M. \& Brown, N. J. (2007) Aldosterone and end-organ damage. Clinical Science, Vol.113, pp. 267-278, PMID17683282

Pitt, B. et al. (1999).The effect of spironolactone on morbidity and mortality in patients with severe heart failure. Randomized Aldactone Evaluation Study Investigators. The New England Journal of Medicine, Vol.341, pp. 709-717, PMID 10471456

Pitt, B. et al. (2003). Eplerenone, a selective aldosterone blocker, in patients with left ventricular dysfunction after myocardial infarction. The New England Journal of Medicine, Vol.348, pp. 1309-1321, PMID 12668699

Prospective Studies Collaboration. (2002). Age-specific relevance of usual blood pressure to vascular mortality: a meta-analysis of individual data for one million adults in 61 prospective studies. The Lancet, Vol.360, pp. 1903-1913, PMID 12493255

Rossi, G.P. et al. (2008). Primary aldosteronism: cardiovascular, renal and metabolic implications. Trends in Endocrinology and Metabolism, Vol.19, pp. 88-90, PMID 18314347

Sato, A. et al. (2003). Effectiveness of aldosterone blockade in patients with diabetic nephropathy. Hypertension, Vol41, pp. 64-68, PMID 12511531

Sechi, L.A. et al. (2006). Long-term renal outcomes in patients with primary aldosteronism. JAMA, Vol.295, pp. 2638-2645, PMID 16772627

Sechi, L.A. et al. (2009). Intrarenal hemodynamics in primary aldosteronism before and after treatment. Journal of Clinical Endocrinology and Metabolism, Vol.94, pp. 1191-1197, PMID 19141581

Sechi, L.A. et al. (2010). Cardiovascular and renal damage in primary aldosteronism: outcomes after treatment. American Journal of Hypertension, Vol.23, pp. 1253-1260, PMID 20706195

The Task Force for the Management of Arterial Hypertension of the European Society of Hypertension (ESH) and of the European Society of Cardiology (ESC). (2007). 2007 Guidelines for the Management of Arterial Hypertension. Journal of Hypertension, Vol.25, pp. 1105-1187, PMID 17762635

Wolf-Maier, K. et al. (2003). Hypertension prevalence and blood pressure levels in 6 European countries, Canada, and the United States. Journal of the American Medical Association, Vol.289, pp. 2363-2369, PMID 12746359

Zannad, F. et al. (2011). Eplerenone in patients with systolic heart failure and mild symptoms. The New England Journal of Medicine, Vol.364: pp. 11-21, PMID 21073363 


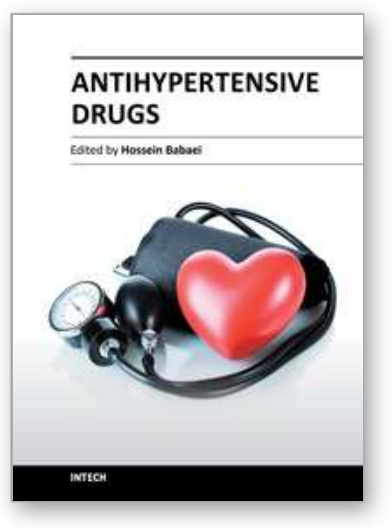

\author{
Antihypertensive Drugs \\ Edited by Prof. Hossein Babaei
}

ISBN 978-953-51-0462-9

Hard cover, 160 pages

Publisher InTech

Published online 28, March, 2012

Published in print edition March, 2012

Hypertension, known as a "silent killer" is widely prevalent and a major risk factor for cardiovascular diseases. It afflicts more than one billion population worldwide and is a leading cause of morbidity and mortality. The authors of the chapters look from different angles to hypertension, sharing their new knowledge and experience in the direction of deep understanding and more clarification of the disease providing an invaluable resource not only for clinicians, but also for all medical sciences students and health providers.

\title{
How to reference
}

In order to correctly reference this scholarly work, feel free to copy and paste the following:

Cristiana Catena, GianLuca Colussi and Leonardo A. Sechi (2012). Potassium-Sparing Diuretics in Hypertension, Antihypertensive Drugs, Prof. Hossein Babaei (Ed.), ISBN: 978-953-51-0462-9, InTech, Available from: http://www.intechopen.com/books/antihypertensive-drugs/potassium-sparing-diuretics-inessential-and-endocrine-hypertension

\section{INTECH}

open science | open minds

\section{InTech Europe}

University Campus STeP Ri

Slavka Krautzeka 83/A

51000 Rijeka, Croatia

Phone: +385 (51) 770447

Fax: +385 (51) 686166

www.intechopen.com

\section{InTech China}

Unit 405, Office Block, Hotel Equatorial Shanghai

No.65, Yan An Road (West), Shanghai, 200040, China 中国上海市延安西路65号上海国际贵都大饭店办公楼 405 单元

Phone: +86-21-62489820

Fax: $+86-21-62489821$ 
(C) 2012 The Author(s). Licensee IntechOpen. This is an open access article distributed under the terms of the Creative Commons Attribution 3.0 License, which permits unrestricted use, distribution, and reproduction in any medium, provided the original work is properly cited. 